\title{
EFETIVIDADE DO TRATAMENTO DE GESTANTES HIPERTENSAS
}

\author{
Mauro Henrique de lima ferrão, Ana Carolina lúcio Pereira, Heloísa Cristina torres Soares Gersgorin, Thales Antônio Abra de Paula, Rosana Rosa
} Miranda Corrêa, Eumenia Costa da Cunha Castro*

Trabalho desenvolvido na Disciplina de Patologia Geral, Departamento de Ciências Biológicas da Universidade Federal do Triângulo Mineiro.

\author{
*Correspondência: \\ Disciplina de Patologia \\ Geral - Departamento de \\ Ciências Biológicas, \\ Universidade Federal do \\ Triângulo Mineiro \\ Avenida Frei Paulino, $n^{\circ} 30$, \\ Bairro Abadia \\ 38025-180, Uberaba, MG \\ Telefone: (34) 3318-5428 \\ Fax: (34) 3318-5462 \\ eumenia.pat@dcb.uftm.edu.br
}

\begin{abstract}
RESUMO
Овјетіvo. Comparar as intercorrências clínicas materno-fetais e a efetividade do tratamento entre grupos das síndromes hipertensivas na gestação (SHG).

Métodos. Foram revisados 200 prontuários de gestantes com SHG, sendo avaliados as intercorrências fetais, a classificação da síndrome hipertensiva e o uso de anti-hipertensivos.

Resultados. Entre as intercorrências maternas, 85 (42,5\%) das pacientes foram classificadas no grupo controle; 32 (16\%) apresentaram hipertensão gestacional (HG); 67 (33,5\%) PE; 6 (3\%) hipertensão crônica; e 10 (5\%) pré-eclâmpsia sobreposta a hipertensão crônica (PSHC). Os menores valores para a idade gestacional, peso dos recém-nascidos e para o índice de Apgar foram observados nos grupos de pacientes com PE e PSHC. A utilização do tratamento não alterou os parâmetros perinatais em relação aos grupos com HG. O grupo de pacientes com PE apresentou a menor idade gestacional e o menor índice de Apgar quando comparado ao grupo controle.
\end{abstract}

CoNcLusÃo. A introdução da terapia anti-hipertensiva durante a gestação foi de fundamental importância para o atendimento à gestante com SHG, embora tenha proporcionado poucos avanços em relação à prevenção das intercorrências perinatais, pois não houve alteração dos parâmetros gestacionais nos casos em que se comparou a utilização do tratamento. A medicação utilizada pouco interfere no fluxo sangüíneo materno-fetal, e conseqüentemente, nas condições de nascimento da criança.

UnItermos: Complicações. Gestação. Hipertensão. Pré-eclâmpsia. Recém-nascido. Tratamento.

\section{INTRODUÇÃO}

As síndromes hipertensivas que ocorrem durante a gestação $(\mathrm{SHG})$ são classificadas em hipertensão crônica $(H C)$, préeclâmpsia/eclâmpsia (PE), pré-eclâmpsia sobreposta à hipertensão crônica (PSHC) e hipertensão gestacional $(H G)$ '. A HG, forma mais freqüente, é relacionada a poucas intercorrências clínicas materno-fetais ${ }^{2}$. A PE pode ocorrer em $5 \%$ das gestações, e suas formas graves ocorrem em 1\%, culminando em crises convulsivas (eclâmpsia) em cerca de $0,05 \%$ dos casos ${ }^{3}$. Essas síndromes ocorrem em 6\% a $8 \%$ das gestações, contribuindo para a prematuridade e morbi-mortalidade perinatal devido à hipóxia intra-uterina ${ }^{4}$.

Entre os fatores de risco para o desenvolvimento das SHG estão a nuliparidade, extremos de idade materna, a cor e a obesidade $^{5}$. Gestantes com hipertensão estão predispostas a desenvolver complicações como o deslocamento prematuro de placenta, coagulação intravascular disseminada, hemorragia cerebral, falência hepática e renal'. A rotura hepática é uma das mais sérias e catastróficas complicações da gravidez, sua incidência varia de l:45.000 a l:225.000 partos, e é usualmente associada à PE. A mortalidade materna é de cerca de $60 \%$ a $86 \%$ e a fetal pode atingir de $56 \%$ a $75 \%$. Entre as complicações fetais está a redução do suprimento de oxigênio e nutrientes ${ }^{6}$, o baixo peso ao nascer ${ }^{7}$ e o maior risco de desenvolver doenças pulmonares agudas e crônicas ${ }^{8}$. Como alterações tardias, crianças pequenas para a idade gestacional, freqüentemente associadas ao diagnóstico de hipertensão gestacional, podem apresentar maiores níveis de pressão arterial e dislipidemia precocemente na fase adulta ${ }^{9}$.

O princípio do tratamento da PE consiste na redução da pressão sangüínea materna e aumento do fluxo sangüíneo placentário. A hidralazina e a metildopa são as drogas usadas comumente como anti-hipertensivos durante a gestação, promovendo o relaxamento do músculo liso das arteríolas periféricas e a redução da resistência vascular ${ }^{10,11}$. Pouco se conhece sobre os efeitos das drogas anti-hipertensivas na circulação placentária ${ }^{10}$. A etiopatogênese da PE envolve uma falha na invasão trofoblástica e não adaptação dos vasos para a gestação, tendo como conseqüência uma redução do fluxo placentário, aumento do espasmo vascular e prejuízo para o equilíbrio hemodinâmico fetal. A redução da resistência de fluxo pela artéria uterina pode possuir efeito positivo na oxigenação e nutrição, melhorando as condições de crescimento fetal. $O$ tratamento com metildopa pode diminuir a resistência vascular uteroplacentária, provavelmente pelo fato das artérias radial e arqueada possuírem inervação adrenérgica'12. No entanto, exames não invasivos para deteç̧ão de alterações na resistência dos vasos uterinos, como a dopplerfluxometria, não demonstram melhora no fluxo sangüíneo fetal em pacientes com PE tratadas com metildopa".

A hipótese deste estudo é que as intercorrências maternofetais e a efetividade do tratamento são diferentes entre os grupos 
de síndromes hipertensivas na gestação. $O$ objetivo do presente trabalho foi avaliar e comparar as intercorrências clínicas maternofetais e a efetividade do tratamento entre grupos das síndromes hipertensivas na gestação avaliados no Hospital Escola da Universidade Federal do Triângulo Mineiro, Uberaba, Minas Gerais.

\section{Métodos}

Este estudo foi aprovado pelo Comitê de Ética em Pesquisa da Universidade Federal do Triângulo Mineiro (UFTM). Foram estudados 200 prontuários de pacientes com partos realizados no Hospital Escola da UFTM, cujas placentas foram enviadas para exame na Disciplina de Patologia Geral no período de 1997 a 2003. A hipertensão arterial foi definida como a pressão sistólica $\geq 140$ $\mathrm{mmHg}$ ou pressão diastólica $\geq 90 \mathrm{mmHg}$, em duas aferições isoladas com intervalo de no máximo uma semana. As síndromes hipertensivas foram classificadas em HC: definida como a hipertensão que está presente e foi observada antes da gestação, ou que foi diagnosticada antes da $20^{\mathrm{a}}$ semana de gestação e não se resolveu após 12 semanas do parto; PE: foi a síndrome específica da gestação que ocorreu após a $20^{\mathrm{a}}$ semana. Foi determinada pela elevação da pressão arterial acompanhada de proteinúria (proteína $\geq 0,3$ g na urina de 24 horas). Na ausência de proteinúria, suspeitou-se de PE quando a elevação da pressão arterial foi acompanhada dos seguintes sinais e sintomas: cefaléia, visão turva, epigastralgia, resultados anormais de testes laboratoriais, especificamente plaquetopenia (contagem de plaquetas $<100.000 / \mathrm{mm}^{3}$ ) e valores anormais de enzimas hepáticas (aumento da alanina aminotransferase ou da aspartato aminotransferase); PSHC: presente em mulheres com hipertensão e sem proteinúria no início da gestação ( $<20$ semanas) e que iniciaram proteinúria, e em mulheres com hipertensão e proteinúria prévias ( $<20$ semanas) e que desenvolveram repentinamente aumento da proteinúria, elevação da pressão arterial anteriormente controlada, plaquetopenia e alteração das enzimas hepáticas; HG: apresentaram elevação da pressão arterial pela primeira vez após 20 semanas de gestação, não acompanhada de proteinúria. $O$ não desenvolvimento da PE e a diminuição da pressão arterial até 12 semanas após o parto asseguraram o diagnóstico de hipertensão gestacional'. Foram classificados como grupo controle as pacientes sem alterações clínicas e laboratoriais avaliadas durante o mesmo período. Foram excluídas pacientes com doença auto-imune, diabetes, além de doença vascular materna conhecida, exceto hipertensão. Outros dados foram obtidos nos prontuários, como a idade e a paridade materna, o uso de antihipertensivos durante a gestação (a-metildopa e hidralazina); o sexo, a idade gestacional e o peso da criança; a gestação única ou gemelar e as intercorrências maternas e fetais. As repercussões do tratamento sobre as intercorrências maternas e fetais foram comparadas entre os grupos com HG e PE. As informações foram analisadas utilizando o programa eletrônico SigmaStat ${ }^{\circledR}$ versão 2.0. As variáveis foram testadas para verificar a normalidade, por meio do teste de Kolmogorov-Smirnov e da análise das variâncias. Para os casos com distribuição normal foi utilizado o teste t de Student na comparação entre dois grupos com distribuição normal, e Anova entre mais de dois grupos com a mesma distribuição. Nas distribuições não normais, foram utilizados testes não paramétricos: o teste de Mann-Whitney para dois grupos e o teste de Kruskal-Wallis para mais de dois grupos. Para comparações múltiplas, foram utilizados os testes de Bonferroni e Dunn. Foram consideradas estatisticamente significantes as diferenças em que a probabilidade foi menor que $5 \%(p<0,05)$.

\section{Resultados}

Da análise dos 200 partos realizados no HE-UFTM, a média de idade das mães foi de 23,6 \pm 5,9 anos e da idade gestacional foi de 37,6 \pm 3,5 semanas, sendo 49 (24,5\%) dos recémnascidos considerados prematuros. A média do peso fetal foi de $2827 \pm 832$ g e quatro (2\%) dos recém-nascidos foram considerados pequenos para a idade gestacional. Em relação ao índice de Apgar, 27 (13,5\%) recém-nascidos tiveram índice da Apgar <7 no primeiro e no quinto minuto, e a maioria estava associada à PE materna. Entre as intercorrências maternas, 85 (42,5\%) pacientes foram classificadas no grupo controle; 32 (16\%) apresentaram HG, 67 (33,5\%) PE, 6 (3\%) HC e 10 (5\%) PSHC. Os menores valores para a idade gestacional, peso dos recém-nascidos ao nascimento e para o índice de Apgar no primeiro minuto foram observados nos grupos de pacientes com PE e PSHC, e as diferenças foram estatisticamente significantes (Tabela I).

No grupo de pacientes com hipertensão gestacional, 16 (50\%) não foram submetidas ao tratamento com anti-hipertensivos e 16 (50\%) foram tratadas. A idade gestacional do grupo com HG tratado foi menor que a observada nos grupos controle e HG não tratado $(p=0,033)$. A mesma distribuição foi observada em relação ao peso de nascimento (Tabela 2). O grupo de pacientes com PE apresentou a menor idade gestacional, o menor peso ao nascer e um baixo índice de Apgar no primeiro e no quinto minuto, quando comparado ao grupo controle $(p<0,00$ I) (Tabela 3).

\section{Discussão}

As SHG são responsáveis por altas taxas de mortalidade materna e fetal, sendo doenças hipertensivas crônicas ou induzidas pela gestação. Em nossos dados, a SHG mais freqüente foi a $P E$, seguida pela $H G$. Estes grupos estão entre os tipos de SHG mais freqüentemente descritos na literatura ${ }^{3}$. Os casos com HC e PSHC tiveram uma freqüência variando entre $1 \%$ e $5 \%$, semelhante a outros trabalhos ${ }^{12}$.

Os menores valores para a idade gestacional, peso dos recémnascidos ao nascimento e para o índice de Apgar no primeiro minuto foram observados nos grupos de pacientes com PE e PSHC. Nestes grupos são descritas as principais intercorrências gestacionais. Na mãe, são destacadas as maiores incidências de hipertensão em futuras gestações e alterações agudas com síndrome HELLP'3. Nos casos de 
Tabela I - Distribuição das pacientes dos grupos controle, com hipertensão gestacional (HG), pré-eclâmpia (PE), hipertensão crônica (HC) e pré-eclâmpsia sobreposta à hipertensão crônica em relação a idade gestacional, peso dos recém-nascidos ao nascimento e índice de Apgar

\begin{tabular}{|c|c|c|c|c|c|}
\hline \multirow[t]{2}{*}{ Grupos } & \multirow[t]{2}{*}{$\begin{array}{c}n(\%) \\
200(100)\end{array}$} & $\begin{array}{l}\text { Idade gestacional } \\
\text { (semanas)* }\end{array}$ & $\begin{array}{l}\text { Peso dos recém- } \\
\text { nascidos (gramas)** }\end{array}$ & $\begin{array}{l}\text { Índice de Apgar } \\
\text { no } 1^{\circ} \text { minuto*** }\end{array}$ & $\begin{array}{l}\text { Índice de Apgar no } \\
5^{\circ} \text { minuto**** }\end{array}$ \\
\hline & & \multicolumn{4}{|c|}{ Média (mín-máx) ou $X \pm D P$} \\
\hline $\begin{array}{l}\text { Controle } \\
\mathrm{HG} \\
\mathrm{PE} \\
\mathrm{HC} \\
\mathrm{PSHC}\end{array}$ & $\begin{array}{l}85(42,5) \\
32(16) \\
67(33,5) \\
6(3) \\
10(5)\end{array}$ & $\begin{array}{l}39,3(38,1-40,2) \\
38,1(36,3-40,1) \\
37,3(34,0-39,2) \\
37,8(36,0-38,6) \\
34,8(32,0-38,4)\end{array}$ & $\begin{array}{l}31 \mid 12,5(2877,5-3380,0) \\
2970,0(2592,5-3437,5) \\
2670,0(1892,5-3252,5) \\
2770,0(2480,0-2950,0) \\
1967,5(1450,0-3245,0)\end{array}$ & $\begin{array}{l}8,8 \pm 1,3 \\
8,8 \pm 1,3 \\
7,1 \pm 2,7 \\
7,5 \pm 3,7 \\
6,2 \pm 3,5\end{array}$ & $\begin{array}{l}9,9 \pm 0,5 \\
9,8 \pm 0,5 \\
8,8 \pm 2,3 \\
8,7 \pm 2,3 \\
8,3 \pm 3,1\end{array}$ \\
\hline
\end{tabular}

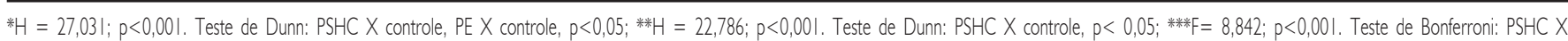
controle, PE X controle $p<0,05 ;$ ****** $F=5,891 ; p<0,001$. Teste de Bonferroni: PSHC X controle

\begin{tabular}{lccc}
\hline \multicolumn{4}{c}{ Tabela 2 - Distribuição das pacientes dos grupos controle e com hipertensão gestacional (HG), tratado e } \\
não tratado, em relaçấo a idade gestacional e peso ao nascimento
\end{tabular}

*H $=6,839 ; p=0,033$. Teste de Dunn: tratado $X$ controle, $p<0,05 ;$ *** $=1,007 ; p=0,369$

Tabela 3 - Distribuição das pacientes dos grupos controle e com pré-eclâmpsia ( $\mathrm{PE})$ em relação a idade gestacional, peso ao nascimento e índice de Apgar no ${ }^{\circ}$ e $5^{\circ}$ minuto

\begin{tabular}{|c|c|c|c|c|c|}
\hline \multirow[b]{2}{*}{ Grupos } & \multirow[b]{2}{*}{$\begin{array}{c}n(\%) \\
140(100)\end{array}$} & \multirow[b]{2}{*}{$\begin{array}{l}\text { Idade gestacional } \\
\text { (semanas)* }\end{array}$} & \multicolumn{2}{|c|}{ Média (mín - máx) ou $X \pm D P$} & \multirow[b]{2}{*}{$\begin{array}{l}\text { Índice de Apgar } \\
5^{\circ} \text { minuto***** }\end{array}$} \\
\hline & & & $\begin{array}{l}\text { Peso ao nascer } \\
\text { (gramas)** }\end{array}$ & $\begin{array}{l}\text { Índice de Apgar } \\
\text { I0minuto**** }\end{array}$ & \\
\hline Controle & $85(42,5)$ & $39,3(38,1-40,2)$ & $3112,5(2877,5-3380,0)$ & $8,8 \pm 1,3$ & $9,9 \pm 0,5$ \\
\hline PE & $67(33,5)$ & $37,3(34,0-39,1)$ & $2670,0(1892,5-3252,5)$ & $7,1 \pm 2,7$ & $8,8 \pm 2,3$ \\
\hline
\end{tabular}

*T $=3619,00 ; p<0,001 ; * * T=4009,000 ; p<0,001 ; * * * T=4,948 ; p<0,001 ; * * * * T=3,907 ; p<0,001$

PSHC, além das complicações da PE, provavelmente há uma alteração vascular materna prévia, provocada pela hipertensão, justificando em parte as intercorrências fetais encontradas, como o menor peso e índice de Apgar.

A menor idade gestacional foi observada nos casos com PE e PSHC Embora existam várias drogas anti-hipertensivas para uso durante a gestação, a interrupção da gestação parece ser a única forma de impedir a evolução das alterações fetais e maternas ${ }^{14}$. Os casos graves exigem interrupção precoce para controle das alterações maternas e do sofrimento fetal, e mesmo os casos de SHG com menores complicações clínicas exigem maiores cuidados que gestações de pacientes normotensas ${ }^{15}$.

Não houve melhora dos padrões perinatais nos casos com PE e HG submetidos ao tratamento. Com os recentes esquemas de tratamento para a hipertensão durante a gestação, a mortalidade materna tem sido reduzida em $0,1 \%$ ", os níveis pressóricos sofrem redução e os riscos para o desenvolvimento de PE são diminuídos ${ }^{16}$. Embora os benefícios maternos sejam claros, pouco se conhece sobre o efeito do tratamento anti-hipertensivo na circulação materno-fetal. A metildopa pode diminuir a resistência vascular uteroplacentária, provavelmente pelo fato das artérias radial e arqueada possuírem inervação adrenérgica' ${ }^{12}$. 0 tratamento com metildopa diminui o espasmo vascular uteroplacentário na artéria uterina, embora não existam diferenças em relação à resistência da artéria umbilical, nem nos parâmetros fetais, como melhora no índice de resistência ao fluxo sangüíneo na ateria cerebral médial".

Estudos demonstram, por meio da avaliação pelo exame de ultra-som com Doppler das artérias umbilicais, arqueada e renal, que 
não há alteração na resistência ao fluxo para a artéria renal materna com o uso de hidralazina, embora a pressão sangüínea materna tenha sido reduzida. Em conseqüência, o fluxo sangüíneo placentário não é afetado pelas doses de hidralazina recomendadas para o consumo oral ${ }^{10}$. Outros estudos afirmam que há redução da resistência vascular uteroplacentária nos casos de SHG tratadas, embora o peso ao nascimento entre estes grupos não tenha alteração ${ }^{12}$. Portanto, mesmo melhorando a circulação local, os benefícios parecem não ser suficientes para alterar as condições de vida do recém-nascido.

Em grupos considerados de menor gravidade materna, como os casos com HG, a introdução da terapia anti-hipertensiva não melhorou aspectos rotineiros usados para a avaliação do neonato, como o peso de nascimento e a idade gestacional. Mesmo não sendo esclarecidas na literatura as diferenças entre a etiopatogênese da hipertensão gestacional e a PE, as SHG parecem reagir de forma semelhante, variando apenas no nível de gravidade, sugerindo mecanismos parecidos para o desenvolvimento do quadro clínico materno. Nos casos com PE, a gravidade das repercussões fetais pode ser comprovada pelas alterações no processo de implantação da placenta, em que a invasão trofoblástica ocorre de forma anômala, fazendo com que as artérias espiraladas não sofram as modificações fisiológicas, principalmente em sua porção miometrial e decidual ${ }^{17}$, diminuindo o fluxo placentário pelo aumento do espasmo vascular e prejudicando o equilíbrio hemodinâmico fetal". Em nossos dados, o menor peso e índice de Apgar dos recém-nascidos foram observados nos grupos de PSHC, sugerindo uma sobreposição de mecanismos responsáveis pelo baixo fluxo sangüíneo. Além das alterações nas arteríolas espiraladas maternas na porção decidual, pacientes com PSHC podem apresentar alterações nos vasos que fazem irrigação dos seguimentos miometriais uterinos, contribuindo para mais prejuízos ao aporte sangüíneo fetal.

\section{ConClusão}

Portanto, mesmo com a introdução da terapia anti-hipertensiva durante a gestação, que sem dúvida foi de fundamental importância para $\mathrm{O}$ atendimento à gestante com $\mathrm{SHG}$, poucos avanços foram conseguidos em relação à prevenção das intercorrências perinatais. A medicação utilizada pouco interfere no fluxo sangüíneo materno-fetal e, conseqüentemente, nas condições de nascimento da criança. Mais estudos são necessários para o entendimento dos mecanismos responsáveis pelo desenvolvimento dos diferentes tipos de SHG na tentativa de melhorar o atendimento pré-natal, bem como o desenvolvimento de terapias capazes de atuar na melhora do fluxo sangüíneo placentário e fetal.

Conflito de interesse: Este trabalho contou com o apoio financeiro da CAPES (Coordenação de Aperfeiçoamento de Pessoal de Nível Superior), CNPq (Conselho Nacional de Desenvolvimento Científico e Tecnológico), FAPEMIG (Fundação de Amparo à Pesquisa de Minas Gerais) e FUNEPU (Fundação de Ensino e Pesquisa de Uberaba).

\section{SUMMARY}

\section{Perinatal outcome in the different clinical forms of HYPERTENSION DURING PREGNANCY}

OBIECTIVE. To compare the maternal-fetal clinical intercurrences and the effectiveness of treatment in the different clinical forms of hypertensive syndromes during pregnancy (HSP).

METHODS. Medical records of 200 pregnant women with HSP were reviewed to appraise fetal intercurrences, classification of the hypertensive syndrome and use of antihypertensives.

RESULTS. Of the 200 patients analyzed, 85 (42.5\%) were controls; 32 (16\%) presented gestational hypertension (GH), 67 (33.5\%) had Pre-eclampsia (PE), 6 (3\%) had chronic hypertension and $10(5 \%)$ cases had PE superimposed chronic hypertension (PSCH). The lowest values for gestational age, weights of the newborn and for the Apgar index were observed in the patients with $P E$ and PSCH. Treatment did not alter the Apgar index in relation to control and non-treated GH patients. Patients with PE presented the lowest gestational age and the smallest Apgar index when compared to controls.

CONCLUSION. Introduction of an antihypertensive therapy during gestation was of fundamental importance for health improvement and pressure control of the pregnant woman with HSP. Nevertheless, it has been of little help for prevention of perinatal intercurrences. This was substantiated by the absence of improvement in the gestational conditions between the treated group when compared to the non-treated. Medication did not significantly improve the maternal-fetal blood flow and consequently in the birth condition of the child. [Rev Assoc Med Bras 2006; 52(6): 390-4]

KEY worDs: Complications. Pregnancy. Hypertension. Pre-eclampsia. Newborn. Treatment.

\section{REFERÊNCIAS}

I. Gifford RW. Report of the National High Blood Pressure Education Program orking Group on High Blood Pressure in Pregnancy. Am J Obstet Gynecol. 2000; 183: I-22.

2. Terrone DA, Rinehart BK, May WL, Martin RW, Martin JN Jr. The myth of transient hypertension: descriptor or disease process? Am J Perinatol. 2001; 18:73-77.

3. Erkkola R. Can pre-eclampsia be predicted and prevented? Acta Obstet Gynecol. Scand. 1997; 164:98-100.

4. Kahhale S; Zugaib M. Conceito, classificação e incidência das síndromes hipertensivas na gestação. HiperAtivo. 1997;4:39-43.

5. Gaio DS, Schmidt MI, Duncan BB, Nucci LB, Matos MC, Branchtein L. Hypertensive disorders in pregnancy: frequency and associated factors in a cohort of Brazilian women. Hypertens Pregnancy. 200I;20:269-8I

6. Lang U, Baker RS, Braems G, Zygmunt M, Kunzel W, Clark KE. Uterine blood flow a determinant of fetal growth. Eur J Obstet Gynecol Reprod Biol. 2003; | | 0:55-61.

7. Xiong X, Demianczuk NN, Saunders LD, Wang FL, Fraser WD. Impact of pregnancy-induced hypertension on fetal growth. Am J Obstet Gynecol. 1999: | 80:207-13. 
8. Gotner L, Hilgendorff A, Bahner T, Ebsen M, Reiss I, Rudloff S. Hypoxiainduced intrauterine growth retardation: effects on pulmonary development and surfactant protein transcription. Biol Neonate. 2005;88: 129-35.

9. Di Cesare Merlone A, Bozzola E, Cerbo RM, Rondini G. Intrauterine growth retardation: diagnostic and therapeutic approach. Minerva Pediatr. 2004;56: I 83-8.

10. Gudmundsson S, Gennser G, Marsal K. Effects of hydralazine on placental and renal circulation in pre-eclampsia. Acta Obstet Gynecol Scand. 1995;74:415-8.

I I. Günenç O, Çiçek N, Gorkemli H, Celik C, Acar A, Akyrek C. The effect of methyldopa treatment on uterine, umbilical and fetal middle cerebral artery blood flows in preeclamptic patients. Arch Gynecol Obstet. 2002;266: | $4 \mid-4$.

12. Rey E. Effects of methyldopa on umbilical and placental artery blood flow velocity waveforms. Obstet Gynecol. 1992;80:783-7.

13. Coppage KH, Sibai BM. Treatment of hypertensive complications in pregnancy. Curr Pharm Des. 2005; 1 1:749-57.
14. Buchbinder A. Adverse perinatal outcomes are significantly higher in severe gestational hypertension than in mild preeclampsia. Am J Obstet Gynecol. 2002; 186:66-7।.

15. Gofton EN, Capewell V, Natale R, Gratton RJ. Obstetrical intervention rates and maternal and neonatal outcomes of women with gestational hypertension. Am J Obstet Gynecol. 200 I ; 185:798-803.

16. Weitz C, Khouzami V, Maxwell K, Johnson JW. Treatment of hypertension in pregnancy with methyldopa: a randomized double blind study. Int J Gynaecol Obstet. 1987;25:35-40.

17. Aguiar RALP, Cabral ACV, Lana AMA. Associação entre a incisura diastólica das artérias uterinas e a histologia do leito placentário em grávidas com pré-eclâmpsia. Rev Bras Ginecol Obstet. 2001 ; 23:43I-38.

Artigo recebido: 25/08/2005

Aceito para publicação: 18/02/2006 